\title{
Nonlinear Impulsive Differential Equations with Weighted Exponential or Ordinary Dichotomous Linear Part in a Banach Space
}

\author{
Hristo Kiskinov and Andrey Zahariev \\ Faculty of Mathematics and Informatics, University of Plovdiv, 236 Bulgaria Boulevard, 4003 Plovdiv, Bulgaria \\ Correspondence should be addressed to Hristo Kiskinov; kiskinov@uni-plovdiv.bg
}

Received 7 July 2015; Accepted 15 September 2015

Academic Editor: Elena Braverman

Copyright (C) 2015 H. Kiskinov and A. Zahariev. This is an open access article distributed under the Creative Commons Attribution License, which permits unrestricted use, distribution, and reproduction in any medium, provided the original work is properly cited.

We consider nonlinear impulsive differential equations with $\psi$-exponential and $\psi$-ordinary dichotomous linear part in a Banach space. By the help of Banach's fixed-point principle sufficient conditions are found for the existence of $\psi$-bounded solutions of these equations on $\mathbb{R}$ and $\mathbb{R}_{+}$.

\section{Introduction}

Impulsive differential equations are an adequate mathematical apparatus for simulation of numerous processes and phenomena in biology, physics, chemistry, control theory, and so forth which during their evolutionary development are subject to short time perturbations in the form of impulses. The qualitative investigation of these processes began with the work of Mil'man and Myshkis [1]. For the first time such equations were considered in an arbitrary Banach space in [2-5].

The problem of $\psi$-boundedness and $\psi$-stability of the solutions of differential equations in finite dimensional Euclidean spaces, introduced for the first time by Akinyele [6], has been studied since then by many authors. A beautiful explanation about the benefits of such a use of weighted stability and boundedness can be found, for example, in [7]. Inspired by the famous monographs of Coppel [8], Daleckii and Krein [9], and Massera and Schaeffer [10], where the important notion of exponential and ordinary dichotomy for ordinary differential equations is considered in detail, Diamandescu [11-13] and Boi [14, 15] introduced and studied the $\psi$-dichotomy for linear differential equations in a finite dimensional Euclidean space, where $\psi$ is a nonnegative continuous diagonal matrix function. The concept of $\psi$ dichotomy for arbitrary Banach spaces was introduced and studied in $[16,17]$. In this case $\psi(t)$ is an arbitrary bounded invertible linear operator.

A weighted dichotomy for linear differential equations with impulse effect in arbitrary Banach spaces is considered in [18] for a $\psi$-exponential dichotomy and in [19] for the particular case of $\psi$-ordinary dichotomy.

This paper considers nonlinear perturbed impulsive differential equations with a $\psi$-dichotomous liner part in an arbitrary Banach space. We will show that some properties of these equations will be influenced by the corresponding $\psi$ dichotomous impulsive homogeneous linear equation. Sufficient conditions for existence of $\psi$-bounded solutions of this equations on $\mathbb{R}$ and $\mathbb{R}_{+}$in case of $\psi$-exponential or $\psi$ ordinary dichotomy are found.

\section{Preliminaries}

Let $X$ be an arbitrary Banach space with norm $|\cdot|$ and identity Id. By $J$ we will denote $\mathbb{R}$ or $\mathbb{R}_{+}=[0, \infty)$ and by $I$ either $\mathbb{Z}$ or $\mathbb{N} \cup\{0\}$.

We consider the nonlinear impulsive differential equation

$$
\begin{aligned}
\frac{\mathrm{d} x}{\mathrm{~d} t} & =A(t) x+F(t, x) \quad\left(t \neq t_{n}\right), \\
x\left(t_{n}^{+}\right) & =Q_{n} x\left(t_{n}\right)+H_{n}\left(x\left(t_{n}\right)\right) \quad(n \in I),
\end{aligned}
$$


where $T=\left\{t_{n}\right\}_{n \in I}$ is a finite or infinite sequence in $J$. We will say that condition (H1) is satisfied if the following conditions hold:

(H 1.1) $A(t)(t \in J)$ is a continuous operator-valued function with values in the Banach space $L B(X)$ of all linear bounded operators acting in $X$ with the norm $\|\cdot\|$.

(H 1.2) $Q_{n} \in L B(X)(n \in I)$.

(H 1.3) The function $F(t, x): J \times X \rightarrow X$ is continuous with respect to $t$.

(H 1.4) $H_{n}: X \rightarrow X(n \in I)$ are continuous operators.

(H 1.5) $t_{n}<t_{n+1}$ and $\lim _{n \rightarrow \pm \infty} t_{n}= \pm \infty(n \in I)$.

Consider the corresponding linear impulsive equation

$$
\begin{aligned}
\frac{\mathrm{d} x}{\mathrm{~d} t} & =A(t) x \quad\left(t \neq t_{n}, n \in I\right), \\
x\left(t_{n}^{+}\right) & =Q_{n} x\left(t_{n}\right) \quad(n \in I) .
\end{aligned}
$$

Definition 1. By a solution of the impulsive equation (1), (2) (or (3), (4)) we will call a function $x(t)$ which for $t \neq t_{n}$ satisfies (1) (or (3)) and for $t=t_{n}$ satisfies condition (2) (or (4)) and is continuous from the left.

As shown, for instance in $[3,4]$, if the operators $Q_{n}$ have bounded inverse ones, then for the impulsive linear equation (3), (4) there exists a Cauchy operator $V(t),(t \in J)$ by means of which each solution $x(t)$ of (3), (4) for which $x(s)=\xi \in X$ has the form

$$
x(t)=V(t) V^{-1}(s) \xi \quad(t, s \in J) .
$$

Let $R L(X)$ be the subspace of all invertible operators in $L B(X)$ whose inverse operators are bounded too. Let $\psi(t)$ : $J \rightarrow R L(X)$ be continuous with respect to $t \in J$ operator function.

Definition 2. A function $u(t): J \rightarrow X$ is said to be $\psi$ bounded on $J$ if $\psi(t) u(t)$ is bounded on $J$.

Let $C_{\psi}(X, T)$ be the space of all $\psi$-bounded on $J$ functions with values in $X$ which are continuous for $t \notin T$, have discontinuities of the first kind for $t \in T$, and are continuous from the left, which is a Banach space with the norm

$$
|\|f\||_{C_{\psi}}=\sup _{t \in J}|\psi(t) f(t)| .
$$

Definition 3. The linear impulsive equation (3), (4) is said to have a $\psi$-exponential dichotomy on $J$ if there exist a pair of mutually complementary projections $P_{1}$ and $P_{2}=\mathrm{Id}-P_{1}$ and positive constants $N_{1}, N_{2}, v_{1}, v_{2}$ such that

$$
\begin{aligned}
&\left\|\psi(t) V(t) P_{1} V^{-1}(s) \psi^{-1}(s)\right\| \leq N_{1} e^{-v_{1}(t-s)} \\
&(s \leq t, s, t \in J), \\
&\left\|\psi(t) V(t) P_{2} V^{-1}(s) \psi^{-1}(s)\right\| \leq N_{2} e^{-v_{2}(s-t)} \\
& \quad(t \leq s, s, t \in J) .
\end{aligned}
$$

Equation (3), (4) is said to have a $\psi$-ordinary dichotomy on $J$ if (7) hold with $v_{1}=v_{2}=0$. In this case we denote $N=$ $\max \left\{N_{1}, N_{2}\right\}$.

Remark 4. For $\psi(t)=\mathrm{Id}$ for all $t \in J$ we obtain the notion exponential and ordinary dichotomy for impulsive differential equations considered in $[3,20,21]$. That is why our main results in this paper appear as a generalization of some results there.

Let us introduce the principal Green function of the nonhomogeneous equation corresponding to (3), (4) with the projections $P_{1}$ and $P_{2}$ from the definition for $\psi$-exponential dichotomy

$$
G(t, s)= \begin{cases}V(t) P_{1} V^{-1}(s) & (t>s, t, s \in J) \\ -V(t) P_{2} V^{-1}(s) & (t<s, t, s \in J) .\end{cases}
$$

Definition 5. The nonnegative function $m(t)$ is said to be integrally bounded on $J$ if for some $l$ the following inequality holds:

$$
B(m(t))=\sup _{t \in J} \int_{t}^{t+l} m(s) \mathrm{d} s<\infty .
$$

Definition 6. The sequence of nonnegative numbers $\left\{m_{n}\right\}_{n \in I}$ is said to be integrally bounded if for some $l$ the following inequality holds:

$$
B\left(m_{j}\right)=\sup _{t \in I} \sum_{t \leq t_{j}<t+l} m_{j}<\infty
$$

For each integrable on $J$ function $m(t)$ we introduce the notation

$$
L(m(t))=\int_{J} m(s) \mathrm{d} s
$$

and for each summable on $I$ sequence of nonnegative numbers $\left\{m_{n}\right\}_{n \in I}$ the notation

$$
L\left(m_{j}\right)=\sum_{j \in I} m_{j}
$$

Definition 7. Let $r>0$ be an arbitrary number. We will say that the function $F(t, x)(t \in J, x \in X)$ and the operators $H_{j}(j \in I)$ satisfy the condition (H2) with the operator function $\psi(t): J \rightarrow R L(X)$, if there exist positive functions $m(t), k(t)(t \in J)$ and sequences of nonnegative numbers $\left\{m_{n}\right\}_{n \in I},\left\{k_{n}\right\}_{n \in I}$ such that

(H 2.1) $|\psi(t) F(t, x)| \leq m(t)(|\psi(t) x| \leq r, t \in J) ;$

$\left(\mathrm{H} \mathrm{2.2)}\left|\psi(t)\left(F\left(t, x_{1}\right)-F\left(t, x_{2}\right)\right)\right| \leq k(t) \mid \psi(t)\left(x_{1}-\right.\right.$ $\left.x_{2}\right) \mid\left(\left|\psi(t) x_{1}\right|,\left|\psi(t) x_{2}\right| \leq r, t \in J\right)$;

(H 2.3) $\left|\psi\left(t_{j}\right) H_{j}(x)\right| \leq m_{j}\left(\left|\psi\left(t_{j}\right) x\right| \leq r, j \in I\right)$;

$\left(\mathrm{H} \mathrm{2.4)}\left|\psi\left(t_{j}\right)\left(H_{j}\left(x_{1}\right)-H_{j}\left(x_{2}\right)\right)\right| \leq k_{j} \mid \psi\left(t_{j}\right)\left(x_{1}-\right.\right.$ $\left.x_{2}\right) \mid\left(\left|\psi\left(t_{j}\right) x_{1}\right|,\left|\psi\left(t_{j}\right) x_{2}\right| \leq r, j \in I\right)$. 
Definition 8. We say that the function $F(t, x)$ and the operators $H_{j}$ belong to the class $E D_{\psi}\left(a_{1}, a_{2}, a_{3}, a_{4}, r\right)$ if the condition ( $\mathrm{H} 2)$ is fulfilled with the operator function $\psi(t)$, the functions $m(t), k(t)$ are integrally bounded on $J$ and $B(m(t)) \leq a_{1}, B(k(t)) \leq a_{2}$, and the sequences $\left\{m_{j}\right\}$ and $\left\{k_{j}\right\}$ are integrally bounded and $B\left(m_{j}\right) \leq a_{3}, B\left(k_{j}\right) \leq a_{4}$.

Definition 9. We say that the function $F(t, x)$ and the operators $H_{j}$ belong to the class $D_{\psi}\left(a_{1}, a_{2}, a_{3}, a_{4}, r\right)$ if the condition $(\mathrm{H} 2)$ is fulfilled with the operator function $\psi(t)$, the functions $m(t), k(t)$ are integrable on $J$ and $L(m(t)) \leq a_{1}, L(k(t)) \leq a_{2}$, and the sequences $\left\{m_{j}\right\}$ and $\left\{k_{j}\right\}$ are summable on $I$ and $L\left(m_{j}\right) \leq a_{3}, L\left(k_{j}\right) \leq a_{4}$.

\section{Main Results}

Theorem 10. Let the following conditions be fulfilled:

(1) The linear impulsive differential equation (3), (4) (i.e., the linear part of (1), (2)) has $\psi$-exponential dichotomy on $\mathbb{R}$ with projections $P_{1}$ and $P_{2}$.

(2) Conditions (H1) and (H2) hold.

(3) The function $F(t, x)$ and the operators $H_{j}$ belong to the class $E D_{\psi}\left(a_{1}, a_{2}, a_{3}, a_{4}, r\right)$.

(4) The operators $Q_{n}$ have bounded inverse ones.

Then for an arbitraryr $>0$, for sufficient small values of $a_{1}$, $a_{2}, a_{3}, a_{4}$ the impulsive equation (1), (2) has a unique solution $x(t)$, which is defined for $t \in \mathbb{R}$ and for which $|\psi(t) x(t)| \leq$ $r(t \in \mathbb{R})$.

Proof. Let $J=\mathbb{R}$. Consider in the space $C_{\psi}(X, T)$ the operator $Q: C_{\psi}(X, T) \rightarrow C_{\psi}(X, T)$ defined by the formula

$$
\begin{aligned}
Q x(t)= & \int_{-\infty}^{\infty} G(t, \tau) F(\tau, x(\tau)) \mathrm{d} \tau \\
& +\sum_{j=-\infty}^{\infty} G\left(t, t_{j}^{+}\right) H_{j}\left(x\left(t_{j}\right)\right),
\end{aligned}
$$

where $G$ is defined by (8).

Now we will show that the ball

$$
S_{\psi, r}=\left\{x:|\|x\||_{C_{\psi}} \leq r\right\}
$$

is invariant with respect to $Q$ and the operator $Q$ is contracting.

First we will prove that the operator $Q$ maps the ball $S_{\psi, r}$ into itself. One has

$$
\begin{aligned}
|\psi(t) Q x(t)| \leq & \left|\int_{-\infty}^{t} \psi(t) G(t, \tau) F(\tau, x(\tau)) \mathrm{d} \tau\right| \\
& +\left|\int_{t}^{\infty} \psi(t) G(t, \tau) F(\tau, x(\tau)) \mathrm{d} \tau\right|
\end{aligned}
$$

$$
\begin{aligned}
& +\left|\sum_{t_{j}<t} \psi(t) G\left(t, t_{j}^{+}\right) H_{j}\left(x\left(t_{j}\right)\right)\right| \\
& +\left|\sum_{t \leq t_{j}} \psi(t) G\left(t, t_{j}^{+}\right) H_{j}\left(x\left(t_{j}\right)\right)\right| .
\end{aligned}
$$

We will estimate the addends in (15). For $x \in S_{\psi, r}$ we obtain

$$
\begin{aligned}
& \left|\int_{-\infty}^{t} \psi(t) G(t, \tau) F(\tau, x(\tau)) \mathrm{d} \tau\right|=\mid \int_{-\infty}^{t} \psi(t) V(t) \\
& \cdot P_{1} V^{-1}(\tau) F(\tau, x(\tau)) \mathrm{d} \tau|=| \int_{-\infty}^{t} \psi(t) V(t) \\
& \cdot P_{1} V^{-1}(\tau) \psi^{-1}(\tau) \psi(\tau) F(\tau, x(\tau)) \mathrm{d} \tau \mid \\
& \leq \int_{-\infty}^{t}\left\|\psi(t) V(t) P_{1} V^{-1}(\tau) \psi^{-1}(\tau)\right\| \mid \psi(\tau) \\
& \cdot F(\tau, x(\tau)) \mid \mathrm{d} \tau \leq N_{1} \int_{-\infty}^{t} e^{-\nu_{1}(t-\tau)} m(\tau) \mathrm{d} \tau \\
& \leq N_{1} \int_{s \leq 0} e^{v_{1} s} m(t+s) \mathrm{d} s \leq N_{1} a_{1} \sum_{k=0}^{\infty} e^{-v_{1} k} \\
& \leq \frac{N_{1} a_{1}}{1-e^{-v_{1}}}, \\
& \left|\int_{t}^{\infty} \psi(t) G(t, \tau) F(\tau, x(\tau)) \mathrm{d} \tau\right|=\mid \int_{t}^{\infty} \psi(t) V(t) \\
& \cdot P_{2} V^{-1}(\tau) F(\tau, x(\tau)) \mathrm{d} \tau|=| \int_{t}^{\infty} \psi(t) V(t) \\
& \cdot P_{2} V^{-1}(\tau) \psi^{-1}(\tau) \psi(\tau) F(\tau, x(\tau)) \mathrm{d} \tau \mid \\
& \leq \int_{t}^{\infty}\left\|\psi(t) V(t) P_{2} V^{-1}(\tau) \psi^{-1}(\tau)\right\| \mid \psi(\tau) \\
& F(\tau, x(\tau)) \mid \mathrm{d} \tau \leq N_{2} \int_{t}^{\infty} e^{-v_{2}(\tau-t)} m(\tau) \mathrm{d} \tau \\
& \leq N_{2} \int_{s \geq 0} e^{-v_{2} s} m(t+s) \mathrm{d} s \leq N_{2} a_{1} \sum_{k=0}^{\infty} e^{-v_{2} k} \\
& \leq \frac{N_{2} a_{1}}{1-e^{-v_{2}}}, \\
& \left|\sum_{t_{j}<t} \psi(t) G\left(t, t_{j}^{+}\right) H_{j}\left(x\left(t_{j}\right)\right)\right|=\mid \sum_{t_{j}<t} \psi(t) V(t) \\
& \cdot P_{1} V^{-1}\left(t_{j}^{+}\right) H_{j}\left(x\left(t_{j}\right)\right)|=| \sum_{t_{j}<t} \psi(t) V(t) \\
& \cdot P_{1} V^{-1}\left(t_{j}^{+}\right) \psi^{-1}\left(t_{j}^{+}\right) \psi\left(t_{j}^{+}\right) H_{j}\left(x\left(t_{j}\right)\right)
\end{aligned}
$$




$$
\begin{gathered}
\leq \sum_{t_{j}<t}\left\|\psi(t) V(t) P_{1} V^{-1}\left(t_{j}^{+}\right) \psi^{-1}\left(t_{j}^{+}\right)\right\| \mid \psi\left(t_{j}\right) \\
\cdot H_{j}\left(x\left(t_{j}\right)\right) \mid \leq N_{1}\left(\sum_{t_{j}<t} e^{\nu_{1}\left(t_{j}-t\right)} m_{j}\right) \leq \frac{N_{1} a_{3}}{1-e^{-v_{1} l}}, \\
\left|\sum_{t \leq t_{j}} \psi(t) G\left(t, t_{j}^{+}\right) H_{j}\left(x\left(t_{j}\right)\right)\right|=\mid \sum_{t \leq t_{j}} \psi(t) V(t) \\
\cdot P_{2} V^{-1}\left(t_{j}^{+}\right) H_{j}\left(x\left(t_{j}\right)\right)|=| \sum_{t \leq t_{j}} \psi(t) V(t) \\
\cdot P_{2} V^{-1}\left(t_{j}^{+}\right) \psi^{-1}\left(t_{j}^{+}\right) \psi\left(t_{j}^{+}\right) H_{j}\left(x\left(t_{j}\right)\right) \mid \\
\leq \sum_{t \leq t_{j}}\left\|\psi(t) V(t) P_{2} V^{-1}\left(t_{j}^{+}\right) \psi^{-1}\left(t_{j}^{+}\right)\right\| \mid \psi\left(t_{j}\right) \\
\cdot H_{j}\left(x\left(t_{j}\right)\right) \mid \leq N_{2}\left(\sum_{t \leq t_{j}} e^{v_{2}\left(t-t_{j}\right)} m_{j}\right) \leq \frac{N_{2} a_{3}}{1-e^{-v_{2} l}} .
\end{gathered}
$$

From the estimates (16) it follows that

$$
\begin{aligned}
|\psi(t) Q x(t)| \leq & \frac{N_{1} a_{1}}{1-e^{-\nu_{1}}}+\frac{N_{2} a_{1}}{1-e^{-\nu_{2}}}+\frac{N_{1} a_{3}}{1-e^{-v_{1} l}} \\
& +\frac{N_{2} a_{3}}{1-e^{-\nu_{2} l}} .
\end{aligned}
$$

Hence by $a_{1} \leq r\left(N_{2} /\left(1-e^{-v_{2}}\right)+N_{1} /\left(1-e^{-\nu_{1}}\right)\right)^{-1}$ and $a_{3} \leq\left(N_{2} /\left(1-e^{-v_{2} l}\right)+N_{1} /\left(1-e^{-v_{1} l}\right)\right)^{-1}$ we obtain

$$
|\psi(t) Q x(t)| \leq r .
$$

Thus the operator $Q$ maps the ball $S_{\psi, r}$ into it self.

Now we will prove that the operator $Q$ is a contraction in the ball $S_{\psi, r}$. Let $x_{1}, x_{2} \in S_{\psi, r}$. Using the same technique as above we obtain

$$
\begin{aligned}
& \left|\psi(t) Q x_{1}(t)-\psi(t) Q x_{2}(t)\right| \leq \int_{-\infty}^{\infty} \mid \psi(t) G(t, \tau) \\
& \cdot\left(F\left(\tau, x_{1}(\tau)\right)-F\left(\tau, x_{2}(\tau)\right)\right)\left|\mathrm{d} \tau+\sum_{j=-\infty}^{\infty}\right| \psi(t) \\
& \cdot G\left(t, t_{j}^{+}\right)\left(H_{j}\left(x_{1}\left(t_{j}\right)\right)-H_{j}\left(x_{2}\left(t_{j}\right)\right)\right) \mid \\
& \leq \int_{-\infty}^{\infty}\left\|\psi(t) G(t, \tau) \psi^{-1}(\tau)\right\| \mid \psi(\tau) \\
& \cdot\left(F\left(\tau, x_{1}(\tau)\right)-F\left(\tau, x_{2}(\tau)\right)\right) \mid \mathrm{d} \tau+\sum_{j=-\infty}^{\infty} \| \psi(t) \\
& \cdot V(t) P_{1} V^{-1}\left(t_{j}^{+}\right) \psi^{-1}\left(t_{j}^{+}\right) \| \mid \psi\left(t_{j}\right)
\end{aligned}
$$

$$
\begin{aligned}
& \cdot\left(H_{j}\left(x_{1}\left(t_{j}\right)\right)-H_{j}\left(x_{2}\left(t_{j}\right)\right)\right)\left|\leq \sup _{\tau \in J}\right| \psi(\tau) \\
& \cdot\left(x_{1}(\tau)-x_{2}(\tau)\right) \mid \int_{-\infty}^{\infty}\left\|\psi(t) G(t, \tau) \psi^{-1}(\tau)\right\| \\
& \cdot k(\tau) \mathrm{d} \tau+\sup _{\tau \in J}\left|\psi(\tau)\left(x_{1}(\tau)-x_{2}(\tau)\right)\right| \\
& \cdot \sum_{j=-\infty}^{\infty}\left\|\psi(t) V(t) P_{1} V^{-1}\left(t_{j}^{+}\right) \psi^{-1}\left(t_{j}^{+}\right)\right\| k_{j} \\
& \leq \sup _{\tau \in J}\left|\psi(\tau)\left(x_{1}(\tau)-x_{2}(\tau)\right)\right|\left(\frac{N_{2} a_{2}}{1-e^{-\nu_{2}}}+\frac{N_{1} a_{2}}{1-e^{-\nu_{1}}}\right. \\
& \left.+\frac{N_{2} a_{4}}{1-e^{-v_{2} l}}+\frac{N_{1} a_{4}}{1-e^{-v_{1} l}}\right) .
\end{aligned}
$$

Hence

$$
\begin{aligned}
& \mid\left\|Q x_{1}-Q x_{2}\right\|_{C_{\psi}} \\
& \quad \leq\left(\frac{N_{2} a_{2}}{1-e^{-\nu_{2}}}+\frac{N_{1} a_{2}}{1-e^{-\nu_{1}}}+\frac{N_{2} a_{4}}{1-e^{-\nu_{2} l}}+\frac{N_{1} a_{4}}{1-e^{-v_{1} l}}\right)\left|\left\|x_{1}-x_{2}\right\|\right|_{C_{\psi}} .
\end{aligned}
$$

Thus by $a_{2}<\left(N_{2} /\left(1-e^{-v_{2}}\right)+N_{1} /\left(1-e^{-v_{1}}\right)\right)^{-1}$ and $a_{4}<$ $\left(N_{2} /\left(1-e^{-v_{2} l}\right)+N_{1} /\left(1-e^{-v_{1} l}\right)\right)^{-1}$ the operator $Q$ is a contraction in the ball $S_{\psi, r}$.

From Banach's fixed point principle, the existence of a unique fixed point of the operator $Q$ follows.

It is not hard to verify that each solution of the impulsive differential equation (1), (2) which lies in the ball $S_{\psi, r}$ is also a solution of the equation

$$
x(t)=Q x(t)
$$

and vice versa.

Corollary 11. If the conditions of Theorem 10 are fulfilled and if, moreover, $F(t, 0)=0(t \in \mathbb{R})$, then $x=0$ is a unique solution of (1), (2) in $C_{\psi}(X, T)$.

Theorem 12. Let the following conditions be fulfilled:

(1) The linear impulsive differential equation (3), (4) (i.e., the linear part of (1), (2)) has $\psi$-exponential dichotomy on $\mathbb{R}$ with projections $P_{1}$ and $P_{2}$.

(2) Conditions (H1) and (H2) hold.

(3) The function $F(t, x)$ and the operators $H_{j}$ belong to the class $D_{\psi}\left(a_{1}, a_{2}, a_{3}, a_{4}, r\right)$.

(4) The operators $Q_{n}$ have bounded inverse ones.

Then for an arbitrary $r>0$, for sufficient small values of $a_{1}$, $a_{2}, a_{3}, a_{4}$ the impulsive equation (1), (2) has a unique solution $x(t)$, which is defined for $t \in \mathbb{R}$ and for which $|\psi(t) x(t)| \leq$ $r(t \in \mathbb{R})$.

Proof. Let $J=\mathbb{R}$. In the proof of Theorem 10 it was mentioned that each solution $x(t)$ of the impulsive differential equation 
(1), (2) that remains for $t \in J$ in the ball $S_{\psi, r}$ satisfies the equation

$$
\begin{aligned}
x(t)= & \int_{-\infty}^{\infty} G(t, \tau) F(\tau, x(\tau)) \mathrm{d} \tau \\
& +\sum_{j=-\infty}^{\infty} G\left(t, t_{j}^{+}\right) H_{j}\left(x\left(t_{j}\right)\right)
\end{aligned}
$$

and vice versa.

We consider again in the space $C_{\psi}(X, T)$ the operator $Q$ : $C_{\psi}(X, T) \rightarrow C_{\psi}(X, T)$ defined in (13). For $|\psi(t) Q x(t)|$ we obtain the following estimate:

$$
\begin{aligned}
& |\psi(t) Q x(t)| \leq \int_{-\infty}^{\infty}|\psi(t) G(t, \tau) F(\tau, x(\tau))| \mathrm{d} \tau \\
& +\sum_{j=-\infty}^{\infty}\left|\psi(t) G\left(t, t_{j}^{+}\right) H_{j}\left(x\left(t_{j}\right)\right)\right| \leq \int_{-\infty}^{\infty} \mid \psi(t) \\
& \cdot G(t, \tau) \psi^{-1}(\tau) \psi(\tau) F(\tau, x(\tau))\left|\mathrm{d} \tau+\sum_{j=-\infty}^{\infty}\right| \psi(t) \\
& \cdot V(t) P_{1} V^{-1}\left(t_{j}^{+}\right) \psi^{-1}\left(t_{j}^{+}\right) \psi\left(t_{j}^{+}\right) H_{j}\left(x\left(t_{j}\right)\right) \mid \\
& \quad \leq \int_{-\infty}^{\infty}\left\|\psi(t) G(t, \tau) \psi^{-1}(\tau)\right\||\psi(\tau) F(\tau, x(\tau))| \mathrm{d} \tau \\
& +\sum_{j=-\infty}^{\infty}\left\|\psi(t) V(t) P_{1} V^{-1}\left(t_{j}^{+}\right) \psi^{-1}\left(t_{j}^{+}\right)\right\| \mid \psi\left(t_{j}\right) \\
& +H_{j}\left(x\left(t_{j}\right)\right)\left|\leq N \int_{-\infty}^{\infty}\right| \psi(\tau) F(\tau, x(\tau)) \mid \mathrm{d} \tau \\
& \left.+a_{3}\right) . \\
& +N \sum_{j=-\infty}^{\infty}\left|\psi\left(t_{j}\right) H_{j}\left(x\left(t_{j}\right)\right)\right|=N \int_{-\infty}^{\infty} m(\tau) \mathrm{d} \tau \\
& +m_{j}=N\left(L\left(m^{\infty}(t)\right)+L\left(m_{j}\right)\right) \leq N\left(a_{1}\right. \\
& \left.+\sum_{j}\right)
\end{aligned}
$$

Thus by sufficiently small $a_{1}$ and $a_{3}$ the operator $Q$ maps the ball $S_{\psi, r}$ into it self.

Now we will prove that the operator $Q$ is a contraction in the ball $S_{\psi, r}$. Let $x_{1}, x_{2} \in S_{\psi, r}$. We obtain

$$
\begin{gathered}
\left|\psi(t) Q x_{1}(t)-\psi(t) Q x_{2}(t)\right| \leq \int_{-\infty}^{\infty} \mid \psi(t) G(t, \tau) \\
\cdot\left(F\left(\tau, x_{1}(\tau)\right)-F\left(\tau, x_{2}(\tau)\right)\right)\left|\mathrm{d} \tau+\sum_{j=-\infty}^{\infty}\right| \psi(t) \\
\cdot G\left(t, t_{j}^{+}\right)\left(H_{j}\left(x_{1}\left(t_{j}\right)\right)-H_{j}\left(x_{2}\left(t_{j}\right)\right)\right) \mid \\
\leq \int_{-\infty}^{\infty}\left\|\psi(t) G(t, \tau) \psi^{-1}(\tau)\right\| \mid \psi(\tau)
\end{gathered}
$$

$$
\begin{aligned}
& \cdot\left(F\left(\tau, x_{1}(\tau)\right)-F\left(\tau, x_{2}(\tau)\right)\right) \mid \mathrm{d} \tau+\sum_{j=-\infty}^{\infty} \| \psi(t) \\
& \cdot V(t) P_{1} V^{-1}\left(t_{j}^{+}\right) \psi^{-1}\left(t_{j}^{+}\right) \| \mid \psi\left(t_{j}\right) \\
& \cdot\left(H_{j}\left(x_{1}\left(t_{j}\right)\right)-H_{j}\left(x_{2}\left(t_{j}\right)\right)\right)\left|\leq N \sup _{\tau \in J}\right| \psi(\tau) \\
& \cdot\left(x_{1}(\tau)-x_{2}(\tau)\right) \mid\left(\int_{-\infty}^{\infty} k(\tau) \mathrm{d} \tau+\sum_{j=-\infty}^{\infty} k_{j}\right) \leq N \\
& \cdot \sup _{\tau \in J}\left|\psi(\tau)\left(x_{1}(\tau)-x_{2}(\tau)\right)\right|\left(a_{2}+a_{4}\right) .
\end{aligned}
$$

Hence

$$
\left.\left\|Q x_{1}-Q x_{2}\right\|\right|_{C_{\psi}} \leq N\left(a_{2}+a_{4}\right)\left|\left\|x_{1}-x_{2}\right\|\right|_{C_{\psi}} .
$$

Thus by sufficiently small $a_{2}$ and $a_{4}$ the operator $Q$ is a contraction in the ball $S_{\psi, r}$.

From Banach's fixed point principle follows the existence of a unique fixed point of the operator $Q$.

Theorem 13. Let the following conditions be fulfilled:

(1) The linear impulsive differential equation (3), (4) (i.e., the linear part of (1), (2)) has $\psi$-exponential dichotomy on $\mathbb{R}$ with projections $P_{1}$ and $P_{2}$.

(2) Conditions (H1) and (H2) hold.

(3) The function $F(t, x)$ and the operators $H_{j}$ belong to the class $E D_{\psi}\left(a_{1}, a_{2}, a_{3}, a_{4}, r\right)$

(4) The operators $Q_{n}$ have bounded inverse ones.

Then for any $r>0$ by sufficient small $a_{1}, a_{2}, a_{3}, a_{4}$ there exists $\rho<r$ such that the impulsive equation (1), (2) has for each $\xi \in X_{1}=P_{1} X$ with $|\psi(0) \xi| \leq \rho$ a unique solution $x(t)$ on $R_{+}$for which $P_{1} x(0)=\xi$ and $|\psi(t) x(t)| \leq r\left(t \in \mathbb{R}_{+}\right)$.

Proof. Let $J=\mathbb{R}_{+}$and $x(t)$ be a solution of (1), (2) that remains for $t \in J$ in the ball $S_{\psi, r}=\left\{x:|\|x\||_{C_{\psi}} \leq r\right\}$. From the results obtained in [18, Theorem 1 and Remark 2] it follows that such $x(t)$ satisfies the integral equation

$$
\begin{aligned}
x(t)= & V(t) \xi+\int_{-\infty}^{\infty} G(t, \tau) F(\tau, x(\tau)) \mathrm{d} \tau \\
& +\sum_{j=-\infty}^{\infty} G\left(t, t_{j}^{+}\right) H_{j}\left(x\left(t_{j}\right)\right),
\end{aligned}
$$

where $\xi=P_{1} x(0)$. The converse is also true: a solution of the (26) satisfies the differential equation (1), (2) for $t \in J$.

Let $\xi \in X_{1}$ and $|\psi(0) \xi| \leq \rho<r$. We consider in the space $C_{\psi}(X, T)$ the operator $Q: C_{\psi}(X, T) \rightarrow C_{\psi}(X, T)$ defined by the formula

$$
\begin{aligned}
Q x(t)= & V(t) \xi+\int_{-\infty}^{\infty} G(t, \tau) F(\tau, x(\tau)) \mathrm{d} \tau \\
& +\sum_{j=-\infty}^{\infty} G\left(t, t_{j}^{+}\right) H_{j}\left(x\left(t_{j}\right)\right) .
\end{aligned}
$$


First we will prove that the operator $Q$ maps the ball $S_{\psi, r}$ into it self. Indeed one has

$$
\begin{gathered}
|\psi(t) Q x(t)| \leq|\psi(t) V(t) \xi|+\mid \psi(t) \\
\cdot\left(\int_{-\infty}^{\infty} G(t, \tau) F(\tau, x(\tau)) \mathrm{d} \tau\right. \\
\left.\quad+\sum_{j=-\infty}^{\infty} G\left(t, t_{j}^{+}\right) H_{j}\left(x\left(t_{j}\right)\right)\right) \mid .
\end{gathered}
$$

For the first addend with $\rho \leq r / 2 N_{1}$ we obtain

$$
|\psi(t) V(t) \xi| \leq N_{1} e^{-v_{1} t}|\psi(0) \xi| \leq N_{1} e^{-v_{1} t} \rho \leq \frac{r}{2}
$$

Using the same technique as in the proof of Theorem 10 we obtain for the second addend the estimate

$$
\begin{aligned}
& \mid \psi(t)\left(\int_{-\infty}^{\infty} G(t, \tau) F(\tau, x(\tau)) \mathrm{d} \tau\right. \\
& \left.\quad+\sum_{j=-\infty}^{\infty} G\left(t, t_{j}^{+}\right) H_{j}\left(x\left(t_{j}\right)\right)\right) \mid \leq \frac{N_{1} a_{1}}{1-e^{-\nu_{1}}} \\
& +\frac{N_{2} a_{1}}{1-e^{-v_{2}}}+\frac{N_{1} a_{3}}{1-e^{-\nu_{1} l}}+\frac{N_{2} a_{3}}{1-e^{-v_{2} l}} .
\end{aligned}
$$

Hence by $a_{1} \leq(r / 2)\left(N_{2} /\left(1-e^{-v_{2}}\right)+N_{1} /\left(1-e^{-v_{1}}\right)\right)^{-1}$ and $a_{3} \leq(r / 2)\left(N_{2} /\left(1-e^{-v_{2} l}\right)+N_{1} /\left(1-e^{-v_{1} l}\right)\right)^{-1}$ we obtain

$$
|\psi(t) Q x(t)| \leq r .
$$

Thus the operator $Q$ maps the ball $S_{\psi, r}$ into it self.

Now we will prove that the operator $Q$ is a contraction in the ball $S_{\psi, r}$. Let $x_{1}, x_{2} \in S_{\psi, r}$. We obtain as in the proof of Theorem 10 the estimate

$$
\begin{aligned}
& \left\|Q Q x_{1}-Q x_{2}\right\|_{C_{\psi}} \\
& \quad \leq\left(\frac{N_{2} a_{2}}{1-e^{-\nu_{2}}}+\frac{N_{1} a_{2}}{1-e^{-\nu_{1}}}+\frac{N_{2} a_{4}}{1-e^{-v_{2} l}}+\frac{N_{1} a_{4}}{1-e^{-\nu_{1} l}}\right)\left|\left\|x_{1}-x_{2}\right\|\right|_{C_{\psi}} .
\end{aligned}
$$

By $a_{2}<\left(N_{2} /\left(1-e^{-v_{2}}\right)+N_{1} /\left(1-e^{-v_{1}}\right)\right)^{-1}$ and $a_{4}<\left(N_{2} /(1-\right.$ $\left.\left.e^{-v_{2} l}\right)+N_{1} /\left(1-e^{-v_{1} l}\right)\right)^{-1}$ the operator $Q$ is a contraction in the ball $S_{\psi, r}$.

From Banach's fixed point principle the existence of a unique fixed point of the operator $Q$ follows.

Theorem 14. Let the following conditions be fulfilled:

(1) The linear impulsive differential equation (3), (4) (i.e., the linear part of (1), (2)) has $\psi$-exponential dichotomy on $\mathbb{R}$ with projections $P_{1}$ and $P_{2}$.

(2) Conditions (H1) and (H2) hold.

(3) The function $F(t, x)$ and the operators $H_{j}$ belong to the class $D_{\psi}\left(a_{1}, a_{2}, a_{3}, a_{4}, r\right)$.

(4) The operators $Q_{n}$ have bounded inverse ones.
Then for any $r>0$ by sufficient small $a_{1}, a_{2}, a_{3}, a_{4}$ there exists $\rho<r$ such that the impulsive equation (1), (2) has for each $\xi \in X_{1}=P_{1} X$ with $|\psi(0) \xi| \leq \rho$ a unique solution $x(t)$ on $R_{+}$for which $P_{1} x(0)=\xi$ and $|\psi(t) x(t)| \leq r\left(t \in \mathbb{R}_{+}\right)$.

Proof. Let $J=\mathbb{R}_{+}, \xi \in X_{1}$, and $|\psi(0) \xi| \leq \rho<r$. We consider again in the space $C_{\psi}(X, T)$ the operator $Q: C_{\psi}(X, T) \rightarrow$ $C_{\psi}(X, T)$ defined by formula (27).

First we will prove that the operator $Q$ maps the ball $S_{\psi, r}$ into it self. One has

$$
\begin{gathered}
|\psi(t) Q x(t)| \leq|\psi(t) V(t) \xi|+\mid \psi(t) \\
\cdot\left(\int_{-\infty}^{\infty} G(t, \tau) F(\tau, x(\tau)) \mathrm{d} \tau\right. \\
\left.\quad+\sum_{j=-\infty}^{\infty} G\left(t, t_{j}^{+}\right) H_{j}\left(x\left(t_{j}\right)\right)\right) \mid .
\end{gathered}
$$

For the first addend with $\rho \leq r / 2 N_{1}$ we obtain

$$
|\psi(t) V(t) \xi| \leq N_{1} e^{-v_{1} t}|\psi(0) \xi| \leq N_{1} e^{-\nu_{1} t} \rho \leq \frac{r}{2}
$$

For the second addend with $a_{1}+a_{3} \leq r / 2 N$ as in the proof of Theorem 12 one has

$$
\begin{aligned}
& \mid \psi(t)\left(\int_{-\infty}^{\infty} G(t, \tau) F(\tau, x(\tau)) \mathrm{d} \tau\right. \\
& \left.\quad+\sum_{j=-\infty}^{\infty} G\left(t, t_{j}^{+}\right) H_{j}\left(x\left(t_{j}\right)\right)\right) \mid \leq N\left(a_{1}+a_{3}\right) \leq \frac{r}{2} .
\end{aligned}
$$

Thus the operator $Q$ maps the ball $S_{\psi, r}$ into it self.

Let $x_{1}, x_{2} \in S_{\psi, r}$. As in the proof of Theorem 12 we obtain the estimate

$$
\left.\left\|Q x_{1}-Q x_{2}\right\|\right|_{C_{\psi}} \leq N\left(a_{2}+a_{4}\right)\left|\left\|x_{1}-x_{2}\right\|\right|_{C_{\psi}} .
$$

Hence by $a_{2}+a_{4}<N^{-1}$ the operator $Q$ is a contraction in the ball $S_{\psi, r}$.

From Banach's fixed point principle the existence of a unique fixed point of the operator $Q$ follows.

In the proof of Theorem 13 it was already mentioned that every solution of the impulsive differential equation (1), (2) which lies in the ball $S_{\psi, r}$ fulfills the equality

$$
x(t)=Q x(t)
$$

and vice versa.

\section{Conflict of Interests}

The authors declare that there is no conflict of interests regarding the publication of this paper. 


\section{Acknowledgment}

This research has been partially supported by Plovdiv University NPD Grant NI15-FMI-004.

\section{References}

[1] V. Mil'man and A. Myshkis, "On the stability of motion in the presence of impulses," Siberian Mathematical Journal, vol. 1, no. 2, pp. 233-237, 1960.

[2] D. D. Baĭnov, S. I. Kostadinov, and A. D. Myshkis, "Bounded and periodic solutions of differential equations with impulse effect in a Banach space," Differential and Integral Equations, vol. 1, no. 2, pp. 223-230, 1988.

[3] D. D. Bajnov, S. I. Kostadinov, and P. Zabrejko, "Exponential dichotomy of linear impulsive differential equations in a Banach space," International Journal of Theoretical Physics, vol. 28, no. 7, pp. 797-814, 1989.

[4] P. P. Zabrejko, D. D. Bajnov, and S. Kostadinov, "Characteristic exponents of impulsive differential equations in a Banach space," International Journal of Theoretical Physics, vol. 27, no. 6, pp. 731-743, 1988.

[5] P. P. Zabrejko, D. D. Bajnov, and S. Kostadinov, "Stability of linear equations with impulse effect," Tamkang Journal of Mathematics, vol. 18, no. 4, pp. 57-63, 1987.

[6] O. Akinyele, "On partial stability and boundedness of degree $k$," Atti della Accademia Nazionale dei Lincei. Rendiconti. Classe di Scienze Fisiche, Matematiche e Naturali VIII, vol. 65, no. 6, pp. 259-264, 1978.

[7] S. Hristova and V. Proytcheva, "Weighted exponential stability for generalized delay functional differential equations with bounded delays," Advances in Difference Equations, vol. 2014, article 185, 2014.

[8] W. A. Coppel, Dichotomies in Stability Theory, vol. 629 of Lecture Notes in Mathematics, Springer, Berlin, Germany, 1978.

[9] J. Daleckii and M. Krein, Stability of Solutions of Differential Equations in Banach Space, American Mathematical Society, Providence, RI, USA, 1974.

[10] J. L. Massera and J. J. Schaeffer, Linear Differential Equations and Function Spaces, Academic Press, New York, NY, USA, 1966.

[11] A. Diamandescu, "Note on the $\psi$-boundedness of the solutions of a system of differential equations," Acta Mathematica Universitatis Comenianae, vol. 73, no. 2, pp. 223-233, 2004.

[12] A. Diamandescu, "Existence of $\psi$-bounded solutions for a system of differential equations," Electronic Journal of Differential Equations, no. 63, pp. 1-6, 2004.

[13] A. Diamandescu, "Existence of $\psi$-bounded solutions for nonhomogeneous linear difference equations," Applied Mathematics E-Notes, vol. 10, pp. 94-102, 2010.

[14] P. Boi, "On the $\psi$-dichotomy for homogeneous linear differential equations," Electronic Journal of Differential Equations, no. 40, pp. 1-12, 2006.

[15] P. Boi, "Existence of $\psi$-bounded solutions for nonhomogeneous linear differential equations," Electronic Journal of Differential Equations, no. 52, pp. 1-10, 2007.

[16] A. Georgieva, H. Kiskinov, S. Kostadinov, and A. Zahariev, " $\psi$ Exponential dichotomy for linear differential equations in a Banach space," Electronic Journal of Differential Equations, no. 153, pp. 1-13, 2013.

[17] A. Georgieva, H. Kiskinov, S. Kostadinov, and A. Zahariev, "Existence of solutions of nonlinear differential equations with $\psi$-exponential or $\psi$-ordinary dichotomous linear part in a Banach space," Electronic Journal of Qualitative Differential Equations, no. 2, pp. 1-10, 2014.

[18] H. Kiskinov, S. Kostadinov, A. Zahariev, and S. Cholakov, "Weighted exponential dichotomy of the solutions of linear impulsive differential equations in a Banach space," Rostocker Mathematisches Kolloquium, vol. 69, pp. 3-17, 2014.

[19] S. Zlatev, "Psi-ordinary dichotomy of the solutions of impulse differential equations in a Banach space," International Journal of Pure and Applied Mathematics, vol. 92, no. 4, pp. 609-618, 2014.

[20] D. Bajnov, S. Kostadinov, and P. Zabrejko, "Dichotomy of the solutions of impulse differential equations in a Banach space," Mathematics Reports Toyama University, vol. 12, pp. 159-166, 1989.

[21] D. D. Bainov, S. I. Kostadinov, and P. P. Zabreiko, "Existence of solutions of nonlinear impulsive differential equations with exponentially dichotomous or dichotomous linear part," International Journal of Theoretical Physics, vol. 30, no. 11, pp. 1545$1554,1991$. 


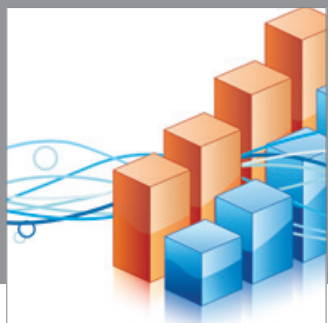

Advances in

Operations Research

mansans

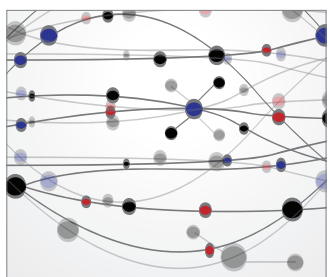

The Scientific World Journal
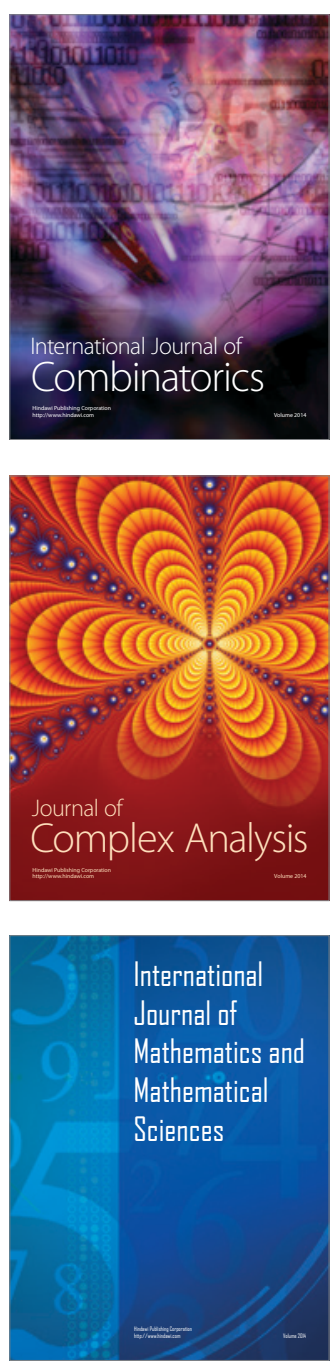
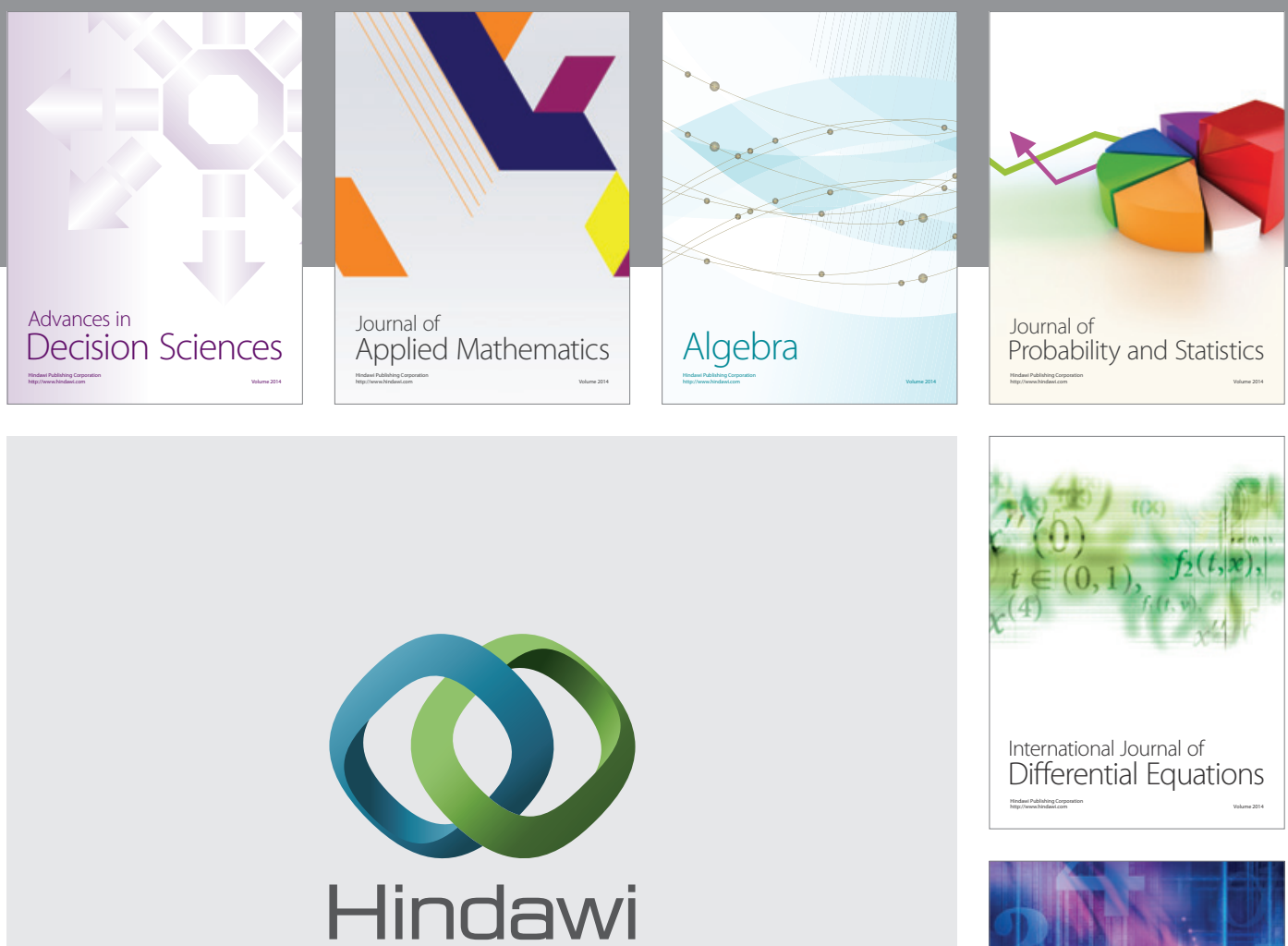

Submit your manuscripts at http://www.hindawi.com
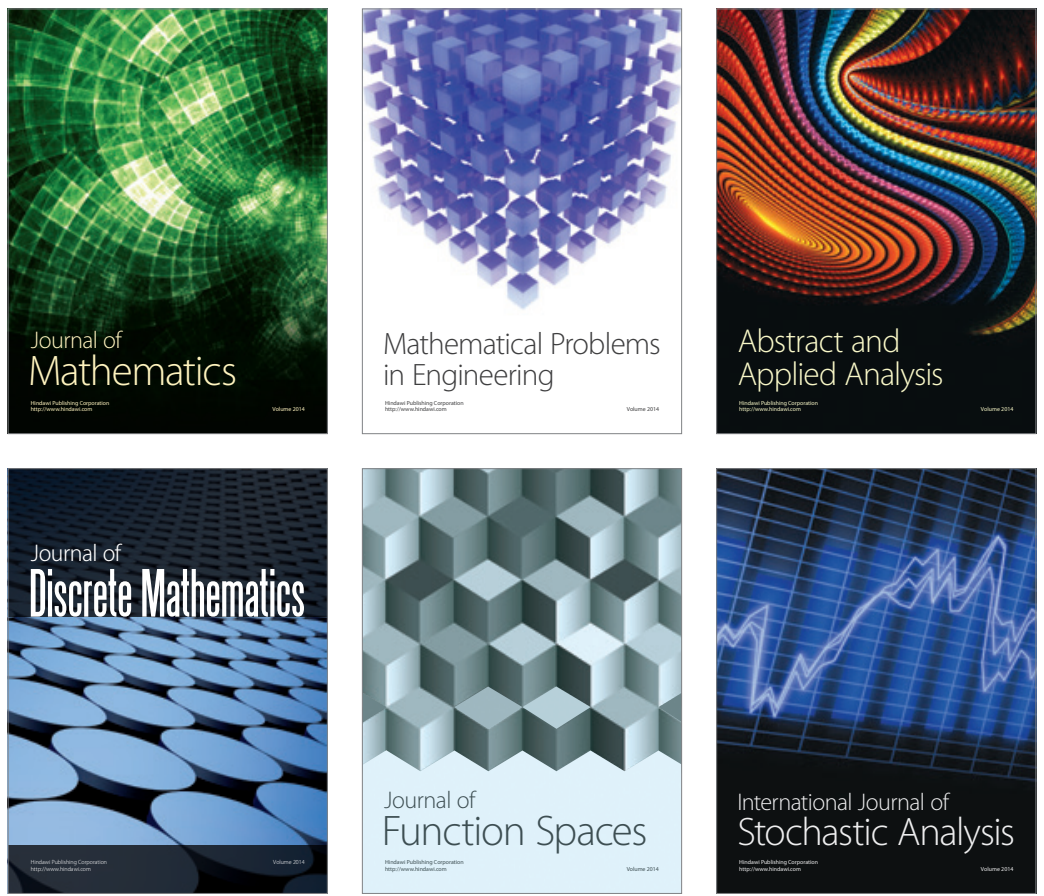

Journal of

Function Spaces

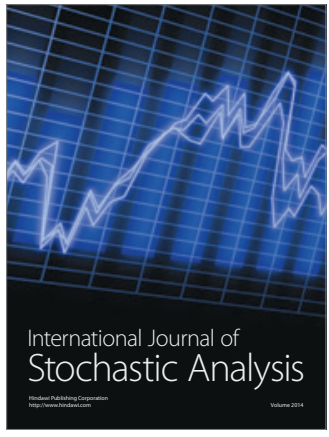

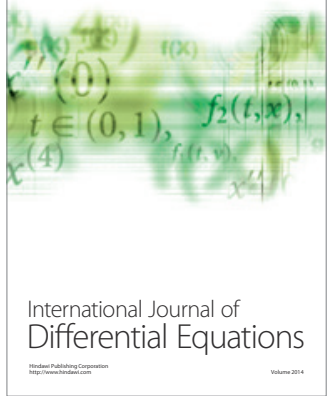
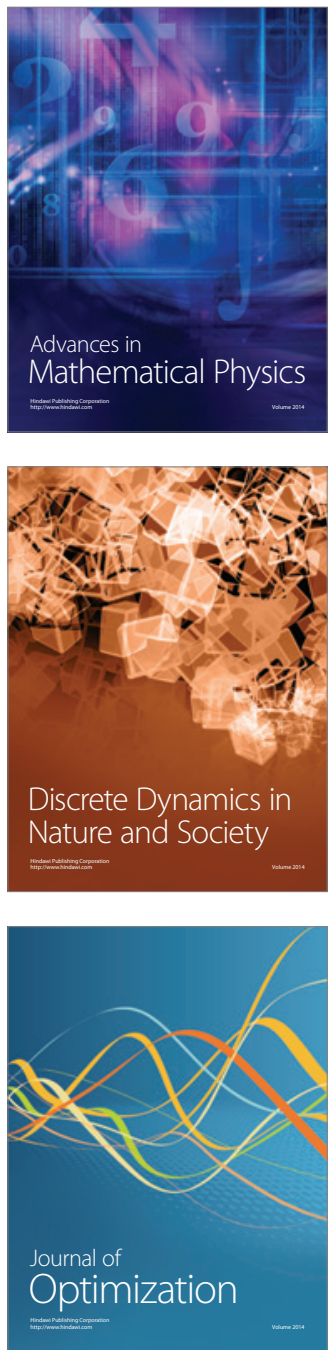